\title{
The facilitators and barriers associated with implementation of a patient-centered medical home in VHA
}

\author{
Christian D. Helfrich ${ }^{1,2^{*}}$, Philip W. Sylling ${ }^{3}$, Randall C. Gale ${ }^{4}$, David C. Mohr ${ }^{5,6}$, Susan E. Stockdale ${ }^{7,8}$, Sandra Joos ${ }^{9}$, \\ Elizabeth J. Brown ${ }^{10,11}$, David Grembowski ${ }^{2}$, Steven M. Asch ${ }^{4,12}$, Stephan D. Fihn 1,3,13, Karin M. Nelson 1,13 \\ and Lisa S. Meredith ${ }^{14,15}$
}

\begin{abstract}
Background: The patient-centered medical home $(P C M H)$ is a team-based, comprehensive model of primary care. When effectively implemented, PCMH is associated with higher patient satisfaction, lower staff burnout, and lower hospitalization for ambulatory care-sensitive conditions. However, less is known about what factors contribute to (or hinder) PCMH implementation.

We explored the associations of specific facilitators and barriers reported by primary care employees with a previously validated, clinic-level measure of PCMH implementation, the Patient Aligned Care Team Implementation Progress Index $\left(\mathrm{Pi}^{2}\right)$.

Methods: We used a 2012 survey of primary care employees in the Veterans Health Administration to perform cross-sectional, respondent-level multinomial regressions. The dependent variable was the $\mathrm{Pi}^{2}$ categorized as high implementation (top decile, 54 clinics, 235 respondents), medium implementation (middle eight deciles, 547 clinics, 4537 respondents), and low implementation (lowest decile, 42 clinics, 297 respondents) among primary care clinics. The independent variables were ordinal survey items rating 19 barriers to patient-centered care and 10 facilitators of PCMH implementation. For facilitators, we explored clinic $\mathrm{Pi}^{2}$ score decile both as a function of respondent-reported availability of facilitators and of rating of facilitator helpfulness.
\end{abstract}

Results: The availability of five facilitators was associated with higher odds of a respondent's clinic's $\mathrm{Pi}^{2}$ scores being in the highest versus lowest decile: teamlet huddles $(O R=3.91)$, measurement tools $(O R=3.47)$, regular team meetings $(\mathrm{OR}=2.88)$, information systems $(\mathrm{OR}=2.42)$, and disease registries $(\mathrm{OR}=2.01)$. The helpfulness of four facilitators was associated with higher odds of a respondent's clinic's $\mathrm{Pi}^{2}$ scores being in the highest versus lowest decile. Six barriers were associated with significantly higher odds of a respondent's clinic's $\mathrm{Pi}^{2}$ scores being in the lowest versus highest decile, with the strongest associations for the difficulty recruiting and retaining providers $(\mathrm{OR}=2.37)$ and non-provider clinicians $(\mathrm{OR}=2.17)$. Results for medium versus low $\mathrm{Pi}^{2}$ score clinics were similar, with fewer, smaller significant associations, all in the expected direction.

Conclusions: A number of specific barriers and facilitators were associated with PCMH implementation, notably recruitment and retention of clinicians, team huddles, and local education. These findings can guide future research, and may help healthcare policy makers and leaders decide where to focus attention and limited resources.

\footnotetext{
* Correspondence: christian.helfrich@va.gov

${ }^{1}$ Seattle-Denver Center of Innovation for Veteran-Centered and Value-Driven Care, VA Puget Sound, U.S. Department of Veterans Affairs, 1660 Columbian Way, S-152, Seattle, 98108 WA, USA

${ }^{2}$ Department of Health Services, University of Washington School of Public Health, Seattle, WA, USA

Full list of author information is available at the end of the article
} 


\section{Background}

The patient-centered medical home $(\mathrm{PCMH})$ is a model of primary care that seeks to change the current episodic, physician-centric model. PCMH is characterized by empaneled patients cared for by a provider-directed team; a whole-person orientation; emphasis on coordination of care, quality and safety; and enhanced access, such as via electronic and telephone communications [1]. While findings are mixed on whether the PCMH model improves outcomes such as quality of care, patient satisfaction and costs [1-4], the adoption of PCMH models is rapidly increasing among US primary care organizations. In a national survey of medical home initiatives involving payment reform, between 2009 and 2013, $\mathrm{PCMH}$ initiatives increased from 26 initiatives covering approximately 5 million patients to 114 initiatives covering approximately 21 million patients [5].

The factors that foster or hinder PCMH implementation are less clear despite the considerable investments of time, resources, and other support [6]. For example, organizational learning strategies, notably learning collaborative, and practice facilitation (practice-change experts providing on-site assistance in combination with other resources such as access to national consultants in practice economics, health IT, etc.), have been the dominant strategies used, but their effectiveness remains uncertain [4]. Qualitative studies have suggested successful implementation of $\mathrm{PCMH}$ requires the presence of engaged, visible leaders [7], who provide an explicit change strategy [8]; the creation of protected time for panel management; daily team huddles; and explicit work load credit for non-face-to-face modalities for delivering clinical care [9]. On the other hand, standard process improvement practices are infrequently used [10]. To close knowledge gaps about factors contributing to $\mathrm{PCMH}$ implementation, we explored the associations of specific barriers and facilitators with a validated measure of $\mathrm{PCMH}$ implementation in a large, integrated health delivery system implementing a $\mathrm{PCMH}$ model in more than 900 clinics.

\section{Methods}

In 2010, the Veterans Health Administration (VHA) launched the Patient Aligned Care Teams (PACT) initiative to implement a $\mathrm{PCMH}$ model in all VHA primary care clinics simultaneously, over 900 clinics nationally. As part of efforts to evaluate the VHA PCMH initiative, extensive national data were collected in 2012 on clinic-level progress in implementing VHA PCMH and factors facilitating and impeding implementation. We assessed the associations between responses to an employee-based survey about VHA PCMH implementation and a validated measure of overall clinic-level VHA PCMH implementation.

\section{Measures and sample}

Independent variables were obtained from a national, web-based survey fielded to all VHA primary care personnel in May and June 2012. The survey link was distributed via an email from VHA central office leadership to regional network and facility leaders with instructions to distribute to primary care personnel. Because the survey was anonymous and voluntary, the precise denominator is unknown; however, the estimated response rate was $25 \%$. Respondents to the VHA $\mathrm{PCMH}$ survey had very similar demographics with primary care respondents from a general employee survey that was fielded the month prior to the VHA PCMH survey. The general employee survey achieved a $62 \%$ response rate. Tables comparing demographic variables have previously been published, and show that the PCMH sample had a slightly higher proportion of supervisors and slightly fewer African Americans but was not significantly different in terms of employee age, tenure, gender, or other racial or ethnic groups [11]. The present analysis focuses on the 96 clinics with survey responses $(n=532)$ from among the 164 clinics in the top and bottom deciles for VHA PCMH implementation, described further below. Analyses of middle decile clinics are included in the Appendices 1, 2, and 3. The survey methodology has previously been reported [11].

The VHA PCMH survey included items assessing barriers to the delivery of optimal patient-centered care and facilitators of $\mathrm{PCMH}$ implementation. The items were developed in collaboration with five regional Demonstration Laboratories, which were established to evaluate the impact of the $\mathrm{PCMH}$ initiative in VHA and test methods of improvement [12]. The survey also included respondent characteristics such as team role, proportion of time spent working in primary care, and supervisory level.

The independent variables were 19 items assessing the barriers and 10 items assessing the facilitators. The question stem for the 19 barrier items was, "How much, if at all, does each of the following factors limit your ability to provide optimal, patient-centered care for your patients?" The barrier items were preceded by a definition of patient-centered care, "An approach to healthcare that prioritizes the Veteran and their values and partners with them to create a personalized strategy to optimize their health, healing and well-being." Respondents scored each barrier on a 3-point ordinal scale from "Does not limit," to "Limits somewhat," to "Limits a great deal." There was also an "N/A or Don't Know" response option. Barriers included factors such as difficulty accessing specialists, recruiting and retaining providers, and highvolume of Computerized Patient Record System (CPRS) automated alerts (e.g., for contraindicated medications).

The question stem for the 10 facilitator items was, "How helpful are the following [PCMH]-related activities 
or resources?" Respondents scored each facilitator on a four-point ordinal scale from "Very Helpful," "Somewhat Helpful," "Not Helpful," to "Not Available/Not Involved." They could also respond "N/A or Don't Know." Facilitators included local education sessions (e.g., a facility or team level in-service on PCMH to help define team roles and responsibilities of the different team members); measurement tools (e.g., use of patient data to evaluate improvement benchmarks); and information systems (e.g., the Primary Care Management Module is a suite of software tools that can be used by primary care teams to assign patients to teams and generate reports). The dependent variable was a validated composite measure of $\mathrm{PCMH}$ implementation, the Patient Aligned Care Team Implementation Progress Index $\left(\mathrm{Pi}^{2}\right)$, which assesses progress in implementing PCMH within VHA, and incorporates data from surveys of primary care personnel, patient surveys, and administrative data [13]. The $\mathrm{Pi}^{2}$ includes 53 measures mapped to 8 domains representing goals of the initiative related to improving (1) access; (2) continuity; (3) care coordination; (4) comprehensive care; (5) self-management support; (6) patient-centered care and communication; (7) shared decision-making; and (8) team-based care. The $\mathrm{Pi}^{2}$ score is calculated as the number of domains in which the clinic appeared in the top and bottom quartiles for the domain scores, ranging from 8 (all domain scores in the top quartile) to -8 (all domain scores in the bottom quartile). The $\mathrm{Pi}^{2}$ has previously been validated by testing the association of clinics in the highest decile of the $\mathrm{Pi}^{2}$ (a score of +5 to +8 ) versus those in the lowest decile of the $\mathrm{Pi}^{2}$ (a score of -8 to -5 ) using measures of quality of care and outcomes that were expected to be favorably associated with PCMH implementation, including higher patient satisfaction, lower staff burnout, higher quality of care, and lower emergency department visits and hospitalizations for ambulatory care-sensitive conditions [13]. For the present analysis, we compared clinics in the lowest decile $(-8$ to $-5, n=87)$ to the highest decile $(+5$ to $+8, n=77) ; 96$ of the 164 clinics in the high and low groups had one or more survey respondents.

Covariates included respondent characteristics, clinic workload and capacity, and setting. Respondent characteristics included VHA tenure, supervisory level, percentage of work hours spent in primary care teams, and occupation. Clinic workload and capacity included four variables from administrative data: (1) overall clinic average panel size, which was adjusted for type of provider (physician vs. nurse practitioner and physician assistant) and FTE; (2) the degree to which the clinic was over paneled relative to a 1200 patients per provider target panel size ( 0 if panel size $\leq 1200$, the percent that average panel size exceeded 1200 if panel size $>1200$ ) [12]; and (3) clinical "intensity" of the patients in the panel, which was the average Diagnostic Cost Group score. Diagnostic Cost Group score is based on inpatient and outpatient diagnoses using the risk adjustment condition categories used by the Center for Medicare and Medicaid Services [14]. For setting, we adjusted for whether the clinic was community based or located with a VHA medical center, the logarithm of total annual visits, and whether the clinic had an academic-affiliation agreement indicating a teaching facility. The variables were selected because we expected they would reflect variation in local patient demand and workforce availability.

\section{Analyses}

We conducted respondent-level multinomial logistic regressions, in which the dependent variable was the respondent's clinic's $\mathrm{Pi}^{2}$ score, categorized as high (top decile), medium (middle eight deciles), and low (lowest decile), as a function of barriers and facilitators. We ran separate models for each barrier and facilitator and adjusted for respondent characteristics and clinic-level workload and staffing measures in each model, described above.

For facilitators, we constructed two sets of models. In the first, we assessed the associations of facilitator "availability" with $\mathrm{Pi}^{2}$ score (high vs. low). A facilitator was defined as unavailable if the respondent rated it Not Available/Not Involved; otherwise, it was defined as available. We constructed a second set of models based upon respondents' categorization of the facilitators as, Very Helpful versus Not or Somewhat helpful.

For primary analyses, responses that were NA/Don't Know were categorized as missing and excluded from the relevant individual model using a standard listwise deletion approach, eliminating observations with missing values from a given model but including those observations in any models where values were not missing. Because NA/Don't Know responses might actually indicate the absence of a barrier or facilitator (rather than truly missing data), we also conducted secondary analysis where NA/Don't Know values were re-coded as a negative response rather than missing (i.e., not a barrier; facilitator not available; or facilitator not helpful). Because frequent NA/Don't Know responses might be indicative of a respondent who either truly did not know or was passively filling out the survey, we also ran the secondary models excluding respondents with $70 \%$ or more NA/Don't Know on items.

In total, we constructed 39 models: 10 models on facilitators available/not available; 10 models on facilitator helpfulness; 19 models on barriers. For reporting purposes, in facilitator models, low implementation clinics were the referent and in barrier models, high implementation clinics were the referent. To simplify the presentation of data, we focus on findings comparing 
high versus low $\mathrm{Pi}^{2}$ clinics which are presented in the main results, but also include findings for comparisons of medium $\mathrm{Pi}^{2}$ clinics versus low (referent) for facilitators, and low $\mathrm{Pi}^{2}$ clinics versus medium (referent) for barriers in the appendices. Models were cluster-adjusted at the clinic level to allow for correlated responses within clinics. We report adjusted odds ratios and $95 \%$ confidence intervals.

In order to assess potential non-response bias, we compared clinics without survey response to clinics with survey responses on covariates. We also assessed the level of inter-rater agreement on barriers and facilitators within site and within occupation.

Analyses were conducted using SAS Enterprise Guide 6.1.

\section{Results}

The mean number of respondents per clinic was 5.5 respondents and the median was 3 . Over $60 \%$ of the respondents had been employed by VHA 5 years or longer. Forty-two percent (42\%) had some supervisory responsibilities and $88 \%$ worked four-fifths or more of their time in primary care (Table 1). The respondents were approximately equally split among 3 of the 4 occupations: primary care provider (PCP), nurse care manager, or clinical associate, and $12 \%$ of the respondents were administrative clerks.

Comparing the clinics in our sample with clinics excluded due to lack of survey data, the clinics that lack survey data had significantly smaller overall patient panels, lower annual visits, and were more likely to be community-based rather than hospital-based outpatient clinics (Table 2). These clinics were no different in terms of average panel size (i.e., the average number of patients cared for by a given provider), average patient comorbidities, or the proportion of providers with panels that were overcapacity. The proportion of clinics excluded for lack of survey data differed by $\mathrm{Pi}^{2}$ group. For high and middle $\mathrm{Pi}^{2}$ clinics, 70 and $73 \%$ had survey data, respectively. Whereas for low $\mathrm{Pi}^{2}$ clinics, $48 \%$ had survey data. The facilitators that were most frequently available were teamlet huddles (90.7\%), measurement tools $(87.8 \%)$, and local education sessions $(87.7 \%)$, while the least frequently available were quality improvement methods (58.9 \%) and the VHA PCMH toolkit (70.6\%) (Table 3$)$. The facilitators most often rated as very helpful were teamlet huddles (53.4\%), regular team meetings $(44.7 \%)$, and disease registries (32.9\%) (Table 4). The barriers to $\mathrm{PCMH}$ implementation most frequently reported as limiting delivery of patient-centered care were difficulty recruiting and retaining providers (59.5\%), volume of automated clinical reminders (54.0\%), and difficulty recruiting and retaining non-provider clinicians (49.5 \%) (Table 5).
Table 1 Respondent characteristics ( $n=532$, sites $=96$ )

\begin{tabular}{|c|c|}
\hline Covariates & Proportion \\
\hline \multicolumn{2}{|l|}{ Respondent characteristics } \\
\hline \multicolumn{2}{|l|}{ Tenure with VA (\%) } \\
\hline Up to 1 year & $7.5 \%$ \\
\hline Between 1 and 5 years & $29.5 \%$ \\
\hline Between 5 and 10 years & $22.9 \%$ \\
\hline Between 10 and 20 years & $26.9 \%$ \\
\hline Over 20 years & $13.2 \%$ \\
\hline \multicolumn{2}{|l|}{ Supervisory responsibilities } \\
\hline None & $58.3 \%$ \\
\hline Team Leader & $34.3 \%$ \\
\hline Manager & $2.8 \%$ \\
\hline First Line Supervisor & $4.3 \%$ \\
\hline Executive/Senior Executive & $0.4 \%$ \\
\hline \multicolumn{2}{|l|}{ Time spent in primary care } \\
\hline$<20 \%$ & $4.8 \%$ \\
\hline $20-40 \%$ & $1.7 \%$ \\
\hline $41-60 \%$ & $1.2 \%$ \\
\hline $61-80 \%$ & $4.0 \%$ \\
\hline$>80 \%$ & $88.2 \%$ \\
\hline \multicolumn{2}{|l|}{ Occupation (\%) } \\
\hline Primary Care Provider & $31.1 \%$ \\
\hline Nurse Care Manager (RN) & $32.4 \%$ \\
\hline Clinical Associate (LPN, medical assistant) & $24.2 \%$ \\
\hline Administrative Clerk & $12.3 \%$ \\
\hline \multicolumn{2}{|l|}{ Clinic characteristics (respondent-level) } \\
\hline \multicolumn{2}{|l|}{ Clinic workload and capacity } \\
\hline PCPs with panels over-capacity (proportion) & $31.4 \%$ \\
\hline Mean level of over-capacity for these panels & $13.3 \%$ \\
\hline Adjusted panel size (mean number of patients) & 1164 \\
\hline Patient intensity (mean DCG patient average) & 0.60 \\
\hline Annual primary care visits & 36,747 \\
\hline Hospital-based clinic (proportion) & $38.7 \%$ \\
\hline Academic-affiliation agreement & $34.5 \%$ \\
\hline
\end{tabular}

When we assessed the level of concordance within the clinic site, for facilitator availability, the median ICC was 0.14 and ranged from a low of 0.07 (quality improvement methods) to a high of 0.24 (teamlet huddles) (Table 3). For facilitator helpfulness, the median ICC was 0.08 and ranged from a low of 0.05 (new approaches to scheduling) to a high of 0.11 (teamlet huddles) (Table 4). For barriers, the median ICC was 0.09 and ranged from a low of 0.03 (patients have limited VAVHA benefits) to a high of 0.27 (recruiting and retaining providers) (Table 5). 
Table 2 Comparison of clinics with and without survey data stratified by $\mathrm{Pi}^{2}$ category

\begin{tabular}{|c|c|c|c|c|c|c|c|c|c|}
\hline & \multicolumn{3}{|c|}{ Highest $\mathrm{Pi}^{2}$ clinics } & \multicolumn{3}{|c|}{ Middle $\mathrm{Pi}^{2}$ clinics } & \multicolumn{3}{|c|}{ Lowest $\mathrm{Pi}^{2}$ clinics } \\
\hline & Surveys & No surveys & $p$ value & Surveys & No surveys & $p$ value & Surveys & No surveys & $p$ value \\
\hline Number & 54 & 23 & - & 547 & 202 & - & 42 & 45 & - \\
\hline$\%$ of $\mathrm{Pi} 2$ group & $70.1 \%$ & $29.9 \%$ & - & $73.0 \%$ & $27.0 \%$ & - & $48.3 \%$ & $51.7 \%$ & - \\
\hline Mean total patients & 3210.9 & 1914.5 & $<0.001$ & 7148.2 & 3397.0 & $<0.001$ & 7709.6 & 2875.4 & $<0.001$ \\
\hline Mean total annual visits & $12,034.4$ & 7613.0 & $<0.001$ & $24,851.3$ & $11,815.7$ & $<0.001$ & $24,668.5$ & 9903.6 & $<0.001$ \\
\hline Adjusted panel size (mean number of patients) & 1130.9 & 1096.9 & 0.68 & 1117.2 & 1130.1 & 0.57 & 1241.6 & 1328.9 & 0.23 \\
\hline Average DCG of panel & 0.562 & 0.537 & 0.55 & 0.588 & 0.537 & 0.0002 & 0.531 & 0.497 & 0.10 \\
\hline PCPs with panels over-capacity (proportion) & $25.93 \%$ & $13.04 \%$ & 0.22 & $25.51 \%$ & $20.00 \%$ & 0.13 & $33.33 \%$ & $35.90 \%$ & 0.81 \\
\hline VAMC (proportion) & $7.41 \%$ & $0.00 \%$ & $0.99^{*}$ & $23.20 \%$ & $5.13 \%$ & $<0.001$ & $22.50 \%$ & $0.00 \%$ & $0.99^{*}$ \\
\hline
\end{tabular}

${ }^{*} p$ value for this comparison may not be reliable due to there being no VAMCs in the No Surveys group

When we assessed the level of concordance within occupation, for facilitator availability, the median ICC was 0.02 and ranged from a low of 0.01 (3 facilitators) to a high of 0.06 (teamlet huddles) (Table 3). For facilitator helpfulness, the median ICC was 0.02 and ranged from a low of 0.01 (4 facilitators) to a high of 0.04 (PACT toolkit and measurement tools) (Table 4). For barriers, the median ICC was 0.01 and ranged from a low of 0.00 (5 barriers) to a high of 0.05 (clinical reminder volume and delivering opiate therapy) (Table 5).

\section{Facilitators, adjusted analyses}

The availability of five facilitators was associated with higher odds of a respondent's clinic being in the highest versus lowest decile for $\mathrm{Pi}^{2}$ scores (Table 3): teamlet huddles $(\mathrm{OR}=3.91$, CI 1.34-11.44), measurement tools $(\mathrm{OR}=3.47$, CI 1.47-8.16), regular team meetings $(\mathrm{OR}=2.88$, CI 1.31-6.30), information systems

Table 3 Odds of a respondent's clinic having a high- $\mathrm{Pi}^{2}$ (vs. low- $\mathrm{Pi}^{2}$ ) score as a function of reporting facilitators were available or that respondents were involved in facilitator activities

\begin{tabular}{|c|c|c|c|c|c|}
\hline & \multirow[b]{2}{*}{$\%^{a}$} & \multicolumn{2}{|l|}{ ICC } & \multicolumn{2}{|c|}{ High $\mathrm{Pi}^{2}$ vs. low $\mathrm{Pi}^{2}$} \\
\hline & & Clinic & Occup. & $\begin{array}{l}\text { Odds } \\
\text { ratio }\end{array}$ & $95 \% \mathrm{Cl}^{\mathrm{b}}$ \\
\hline Local education session & 87.7 & 0.15 & 0.02 & 1.50 & $(0.77,2.93)$ \\
\hline Learning collaborative & 80.0 & 0.12 & 0.01 & 1.20 & $(0.67,2.14)$ \\
\hline Measurement tools & 87.8 & 0.14 & 0.05 & 3.47 & $(1.47,8.16)$ \\
\hline Teamlet huddles & 90.7 & 0.24 & 0.06 & 3.91 & $(1.34,11.44)$ \\
\hline Regular team meetings & 84.5 & 0.19 & 0.02 & 2.88 & $(1.31,6.30)$ \\
\hline Information systems & 83.3 & 0.18 & 0.02 & 2.42 & $(1.21,4.82)$ \\
\hline New approaches to scheduling & 80.9 & 0.12 & 0.01 & 1.34 & $(0.76,2.34)$ \\
\hline Quality improvement methods & 58.9 & 0.07 & 0.01 & 1.38 & $(0.85,2.25)$ \\
\hline Disease registries & 73.1 & 0.13 & 0.04 & 2.01 & $(1.11,3.65)$ \\
\hline PACT toolkit & 70.6 & 0.11 & 0.01 & 1.57 & $(0.88,2.80)$ \\
\hline
\end{tabular}

The overall percentage of respondents who reported the facilitator was available or that the respondent was involved in facilitator activities

${ }^{b}$ Odds ratios with $95 \%$ confidence intervals that do not cross 1.0 are in italics
$(\mathrm{OR}=2.42$, $\mathrm{CI} 1.21-4.82)$, and disease registries $(\mathrm{OR}=$ 2.01, CI 1.11-2.80).

The helpfulness of four facilitators was associated with higher odds of a respondent's clinic being in the highest versus lowest decile for $\mathrm{Pi}^{2}$ scores (Table 4): local education sessions $(\mathrm{OR}=2.02$, CI 1.13-3.63), information systems $(\mathrm{OR}=1.97, \mathrm{CI} 1.19-3.24)$, disease registries $(\mathrm{OR}=1.90$, CI $1.22-2.96)$, and regular team meetings $(\mathrm{OR}=1.68$, CI 1.05-2.70).

\section{Barriers, adjusted analyses}

For six of the 19 barrier items, reporting that delivery of patient-centered care was limited a great deal by the barrier was associated with higher odds of a respondent's clinic being in the lowest versus highest decile for $\mathrm{Pi}^{2}$ scores (Table 5): difficulty recruiting and retaining providers $(\mathrm{OR}=2.37$, CI 1.29-4.33); difficulty recruiting and retaining non-provider clinicians ( $\mathrm{OR}=2.17$, CI 1.17-4.00); lack of support from clinical leadership $(\mathrm{OR}=2.15$, CI 1.213.82); lack of control over one's schedule $(\mathrm{OR}=1.98$, CI 1.15-3.43); inadequate support for patient behavioral change needs $(\mathrm{OR}=2.15$, CI 1.36-3.38); and recruiting and retaining non-clinicians $(\mathrm{OR}=1.96, \mathrm{CI} 1.09-3.53)$.

\section{Medium $\mathrm{Pi}^{2}$-score clinics versus low}

We also found that the availability of three facilitators was associated with higher odds of the respondent's clinic being in the middle eight deciles versus the lowest, and that the reporting of seven barriers was associated with higher odds of the respondent's clinic being in the lowest $\mathrm{Pi}^{2}$ decile versus the middle eight deciles (Appendix 1). In all cases, these were also significant associations in the comparison of respondents in clinics with the highest versus lowest decile for $\mathrm{Pi}^{2}$ score, and in all cases, the associations were smaller but in the expected direction. There were no significant associations between helpfulness of facilitators and the odds of a respondent's clinic being in the middle eight deciles for $\mathrm{Pi}^{2}$ scores versus in the lowest decile. 
Table 4 Odds of a respondent's clinic having a high- $\mathrm{Pi}^{2}$ (vs. low- $\mathrm{Pi}^{2}$ ) score as a function of reporting facilitators were very helpful for implementation of PACT

\begin{tabular}{|c|c|c|c|c|c|}
\hline & & ICC & & High P & $\mathrm{i}^{2}$ vs. low $\mathrm{Pi}^{2}$ \\
\hline & $\%^{\mathrm{a}}$ & Clinic & Occup. & $\begin{array}{l}\text { Odds } \\
\text { ratio }\end{array}$ & $95 \% \mathrm{Cl}^{\mathrm{b}}$ \\
\hline Local education session & 25.7 & 0.09 & 0.04 & 2.02 & $(1.13,3.63)$ \\
\hline Learning collaborative & 26.0 & 0.06 & 0.02 & 1.50 & $(0.87,2.59)$ \\
\hline Measurement tools & 25.2 & 0.10 & 0.04 & 1.44 & $(0.85,2.43)$ \\
\hline Teamlet huddles & 53.4 & 0.11 & 0.01 & 1.57 & $(0.94,2.61)$ \\
\hline Regular team meetings & 44.7 & 0.07 & 0.01 & 1.68 & $(1.05,2.70)$ \\
\hline Information systems & 30.4 & 0.08 & 0.02 & 1.97 & $(1.19,3.24)$ \\
\hline New approaches to scheduling & 29.3 & 0.05 & 0.01 & 1.70 & $(1.00,2.89)$ \\
\hline Quality improvement methods & 23.5 & 0.06 & 0.03 & 2.00 & $(0.93,4.31)$ \\
\hline Disease registries & 32.9 & 0.09 & 0.01 & 1.90 & $(1.22,2.96)$ \\
\hline PACT toolkit & 24.7 & 0.09 & 0.04 & 1.59 & $(0.85,2.97)$ \\
\hline
\end{tabular}

The overall percentage of respondents who reported the facilitator was very helpful for implementation of PACT

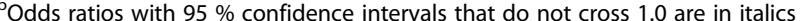

In the secondary analyses testing how robust the results were to different assumptions about NA/Don't Know responses, the results were virtually identical.

\section{Discussion}

To our knowledge, ours is the first paper to explore the associations of specific PCMH facilitators and barriers to patient-centered care with a validated measure of $\mathrm{PCMH}$ implementation across a population of clinics all implementing a PCMH model. This is important because outcomes, such as patient experience and quality of care, differ significantly among clinics depending on how well a PCMH model is implemented $[13,15]$.

We found that a range of facilitators and barriers were significantly associated with a validated measure of $\mathrm{PCMH}$ implementation. The relationships were all in the expected direction, and were frequently large. Respondents within clinics exhibited what organizational scholars generally consider modest (0.08 to 0.12$)$ to high $(>0.20)$ concordance on most items [16], which increases our confidence that surveys accurately captured clinic differences in barriers and facilitators.

Both the availability and ratings of facilitators as very helpful were associated with greater odds of a respondent's

Table 5 Odds of a respondent's clinic having a low- $\mathrm{Pi}^{2}$ (vs. high- $\mathrm{Pi}^{2}$ ) score as a function of reporting that barriers limited delivery of optimal patient-centered care "a great deal"

\begin{tabular}{|c|c|c|c|c|c|}
\hline & & ICC & & Low $\mathrm{Pi}^{2}$ vs. & $\mathrm{Pi}^{2}$ \\
\hline & $\%^{\mathrm{a}}$ & Clinic & Occup. & Odds ratio & $95 \% \mathrm{Cl}^{\mathrm{b}}$ \\
\hline Lack of support from clinical leadership & 34.3 & 0.10 & 0.01 & 2.15 & $(1.21,3.82)$ \\
\hline Difficulty accessing specialist care & 43.1 & 0.14 & 0.01 & 1.52 & $(0.87,2.66)$ \\
\hline Poor communication with specialists within VA & 38.7 & 0.13 & 0.02 & 1.26 & $(0.68,2.35)$ \\
\hline Poor communication with specialists outside the VA & 35.2 & 0.05 & 0.00 & 1.47 & $(0.93,2.31)$ \\
\hline Poor communication around inpatient care & 25.1 & 0.08 & 0.01 & 1.21 & $(0.59,2.47$ \\
\hline Lack of control over my schedule & 34.7 & 0.10 & 0.03 & 1.98 & $(1.15,3.43)$ \\
\hline Lack of responsiveness to my requests for assistance from my team members & 24.0 & 0.06 & 0.01 & 1.65 & $(0.99,2.78)$ \\
\hline Inadequate time allotted to provide counseling or education & 36.9 & 0.05 & 0.01 & 1.24 & $(0.79,1.95)$ \\
\hline Inadequate time allotted to provide follow-up care & 37.3 & 0.09 & 0.01 & 1.59 & $(0.97,2.61)$ \\
\hline Patients have limited VA benefits & 24.2 & 0.03 & 0.02 & 1.59 & $(0.95,2.67)$ \\
\hline Preferred medications are difficult to obtain & 28.4 & 0.10 & 0.00 & 1.21 & $(0.74,2.00)$ \\
\hline Inadequate support for patient behavioral change needs & 26.4 & 0.09 & 0.00 & 2.15 & $(1.36,3.38)$ \\
\hline Recruiting and retaining providers & 59.5 & 0.27 & 0.00 & 2.37 & $(1.29,4.33)$ \\
\hline Recruiting and retaining non-provider clinicians & 49.5 & 0.16 & 0.00 & 2.17 & $(1.17,4.00)$ \\
\hline Recruiting and retaining non-clinicians & 43.0 & 0.10 & 0.01 & 1.96 & $(1.09,3.53)$ \\
\hline Clinical reminder volume & 54.0 & 0.08 & 0.05 & 1.31 & $(0.75,2.27)$ \\
\hline Delivering opiate therapy & 38.9 & 0.08 & 0.04 & 1.55 & $(0.84,2.86)$ \\
\hline Time \& effort to input notes & 35.2 & 0.05 & 0.04 & 1.16 & $(0.66,2.05)$ \\
\hline High volume of Computerized Patient Record System (CPRS) alerts & 41.5 & 0.08 & 0.05 & 1.48 & $(0.79,2.77)$ \\
\hline
\end{tabular}

${ }^{a}$ The overall percentage of respondents who reported the barrier limited delivery of optimal patient-centered care a great deal

${ }^{b}$ Odds ratios with $95 \%$ confidence intervals that do not cross 1.0 are in italics 
clinic being in the highest versus lowest decile for $\mathrm{Pi}^{2}$ score. This suggests that, in 2012, primary care employees at the clinics with the most limited progress on VHA PCMH implementation may have lacked both access to or awareness of resources and may have found that available implementation resources failed to meet their needs.

The facilitators that distinguished high-implementation from low-implementation clinics included factors related to infrastructure, such as disease registries and recruiting and retaining employees, as well as factors related to process, such as reported helpfulness of teamlet huddles and team meetings, and local PCMH education sessions.

These national survey results corroborate findings from prior in-depth evaluations of PCMH implementation at VHA demonstration clinics. At demonstration clinics, data infrastructure was critical for practice redesign to be successful, and to allow for effective panel management [9], and VHA providers who participated in daily huddles reported greater self-efficacy for implementing PCMH changes [17].

Recruiting and retaining providers and non-provider clinicians were both among the most prevalent barriers, and had the strongest associations with PCMH implementation. Respondents at clinics with the lowest $\mathrm{Pi}^{2}$ scores were more likely to report patient-centered care was hindered due to challenges in recruiting and retaining providers, non-provider clinicians and non-clinicians. This is consistent with earlier findings that inadequate staffing of teamlets was a major challenge to making the VHA PCMH model work [18], and that provider turnover had worsened since the launch of the PCMH initiative, with turnover significantly greater for older and more experienced providers [19]. Turnover in PCMH teamlet members likely contributes to other barriers (e.g., difficulties in holding meetings regularly to coordinate work, and lack of responsiveness from team members), which may make turnover among both providers and other team members a particularly important challenge to effectively implementing the new model.

Although clinical reminder volume and electronic medical record system alert volumes were frequently rated by respondents as barriers to delivery of patient-centered care, they were not associated with the odds of a respondent's clinic being in the highest versus lowest decile for $\mathrm{Pi}^{2}$ score. Neither was delivery of opiate therapy which was cited as a major concern in open-text responses from this same survey [18]. A probable explanation is that these barriers were pervasive system-wide, and exhibit limited variation across clinics, and therefore do not differentiate PCMH implementation among clinics. However, due to their prevalence, these barriers may be very important to address, irrespective of whether they are associated with $\mathrm{PCMH}$ implementation.

\section{Limitations}

This was a cross-sectional analysis of observational data. This design is susceptible to unobserved confounding, and we cannot assume that the observed associations are causal. We found potential selection bias in terms of the clinics excluded due to lack of survey data. These clinics tended to be smaller, community-based clinics, and were more likely to be a low- $\mathrm{Pi}^{2}$ clinic. This type of selection bias would most likely increase our risk of a type 2 error, and it is possible that we would have found additional significant associations with a more representative sample of clinics. It is also possible that our findings are due to inferential bias, in which respondents at high-implementation clinics infer that they encountered fewer barriers and received more helpful resources because they observed greater implementation of the VHA PCMH model. However, this type of bias, called representativeness heuristic, usually arises when someone infers an answer to a more complex question (e.g., how much progress have you made on VHA PCMH) from a simpler, specific observation (e.g., have you been able to hire staff), which is the opposite of the present analysis [20]. It is also of greater concern when the respondent is rating both the outcome and the explanatory variables. In this case, the outcome is a clinic-level index incorporating data from a range of sources and respondents completed the survey without knowledge of their clinic's $\mathrm{Pi}^{2}$ score.

We tested 39 associations, and it is possible that significant findings are just chance associations. However, we found a total of 15 significant associations when we would expect to find approximately two by chance $(39 * 0.05=1.95)$. Moreover, the associations were all in the expected direction, and we found a similar but weaker pattern of associations when comparing clinics in the lowest decile for $\mathrm{Pi}^{2}$ score with clinics in the middle eight deciles.

Finally, we used a discrete set of structured measures of barriers and facilitators of $\mathrm{PCMH}$ implementation that may have omitted important factors. Some factors we may have omitted, such as strategies for integrating additional clinic members, for example, pharmacists [21], could play an important role in implementation of the VHA PCMH model. Other potentially omitted factors, such as the effects of healthcare reform and conflicting criteria for $\mathrm{PCMH}$ designation [22], are likely less salient for VHA clinics, but may be vitally important in other settings.

\section{Conclusions}

Overall, our findings suggest that a number of factors may have important influences on PCMH implementation, including structural factors, such as clinical IT and recruitment and retention of clinicians and staff, as well 
as process issues, such as team huddles and quality of local education sessions on PCMH.

Our findings make a modest but important contribution to the literature by exploring associations of key barriers and facilitators with PCMH implementation in a large, national sample of VHA primary care clinics using a validated, structured measure of $\mathrm{PCMH}$ implementation. These findings may help guide future research and may be useful to healthcare policy makers and leaders engaged in PCMH implementation, who must decide where to focus finite attention and devote limited resources.

\section{Appendix 1}

Table 6 Odds of a respondent's clinic being in the middle eight deciles for $\mathrm{Pi}^{2}$ score (vs. lowest decile) as a function of reporting facilitators were available or that respondents were involved in facilitator activities

\begin{tabular}{llc}
\hline & \multicolumn{2}{c}{ Medium $\mathrm{Pi}^{2}$ vs. low $\mathrm{Pi}^{2}$} \\
\cline { 2 - 3 } & Odds ratio & $p$ value \\
\hline Local education session & 0.93 & 0.68 \\
Learning collaborative & 0.99 & 0.93 \\
Measurement tools & 1.44 & 0.03 \\
Teamlet huddles & 1.36 & 0.15 \\
Regular team meetings & 1.27 & 0.14 \\
Information systems & 1.35 & 0.06 \\
New approaches to scheduling & 1.08 & 0.66 \\
Quality improvement methods & 1.24 & 0.16 \\
Disease registries & 1.60 & 0.02 \\
PACT toolkit & 1.01 & 0.96 \\
\hline
\end{tabular}

\section{Appendix 2}

Table 7 Odds of a respondent's clinic being in the middle eight deciles for $\mathrm{Pi}^{2}$ score (vs. lowest decile) as a function of reporting facilitators were very helpful for implementation of PACT

\begin{tabular}{llc}
\hline & \multicolumn{2}{l}{ Medium $\mathrm{Pi}^{2}$ vs. low $\mathrm{Pi}^{2}$} \\
\cline { 2 - 3 } & Odds ratio & $\mathrm{p}$ value \\
\hline Local education session & 1.25 & 0.26 \\
Learning collaborative & 1.17 & 0.43 \\
Measurement tools & 1.14 & 0.51 \\
Teamlet huddles & 1.12 & 0.46 \\
Regular team meetings & 1.30 & 0.10 \\
Information systems & 1.32 & 0.08 \\
New approaches to scheduling & 1.17 & 0.38 \\
Quality improvement methods & 1.13 & 0.64 \\
Disease registries & 1.30 & 0.09 \\
PACT toolkit & 0.98 & 0.92 \\
\hline
\end{tabular}

\section{Appendix 3}

Table $\mathbf{8}$ Odds of a respondent's clinic being in the lowest decile for $\mathrm{Pi}^{2}$ score (vs. middle eight deciles) as a function of reporting that barriers limited delivery of optimal patient-centered care a great deal

\begin{tabular}{|c|c|c|}
\hline & \multicolumn{2}{|c|}{$\begin{array}{l}\text { Low } \mathrm{Pi}^{2} \mathrm{Vs} \\
\text { medium } \mathrm{Pi}^{2}\end{array}$} \\
\hline & $\begin{array}{l}\text { Odds } \\
\text { ratio }\end{array}$ & $p$ value \\
\hline Lack of support from clinical leadership & 1.34 & 0.10 \\
\hline Difficulty accessing specialist care & 1.27 & 0.25 \\
\hline Poor communication with specialists within VA & 1.04 & 0.84 \\
\hline Poor communication with specialists outside the VA & 1.01 & 0.92 \\
\hline Poor communication around inpatient care & 1.14 & 0.46 \\
\hline Lack of control over my schedule & 1.37 & 0.04 \\
\hline $\begin{array}{l}\text { Lack of responsiveness to my requests for assistance } \\
\text { from my team members }\end{array}$ & 1.58 & 0.01 \\
\hline $\begin{array}{l}\text { Inadequate time allotted to provide counseling } \\
\text { or education }\end{array}$ & 1.01 & 0.95 \\
\hline Inadequate time allotted to provide follow-up care & 1.22 & 0.30 \\
\hline Patients have limited VA benefits & 1.42 & 0.05 \\
\hline Preferred medications are difficult to obtain & 1.18 & 0.36 \\
\hline $\begin{array}{l}\text { Inadequate support for patient behavioral } \\
\text { change needs }\end{array}$ & 1.40 & 0.03 \\
\hline Recruiting and retaining providers & 1.56 & 0.05 \\
\hline Recruiting and retaining non-provider clinicians & 1.69 & 0.004 \\
\hline Recruiting and retaining non-clinicians & 1.38 & 0.06 \\
\hline Clinical reminder volume & 1.15 & 0.39 \\
\hline Delivering opiate therapy & 1.08 & 0.65 \\
\hline Time \& effort to input notes & 1.02 & 0.93 \\
\hline CPRS alerts volume & 1.24 & 0.20 \\
\hline
\end{tabular}

\section{Competing interests}

The authors declare that they have no competing interests.

\section{Authors' contributions}

CDH, DCM, SES, SJ, DG, and LSM are members of an evaluation workgroup that developed the survey, and oversaw data collection and analysis. PWS conducted the analyses. SDF and KMN are leaders of the national PACT evaluation that conducted the survey, and were instrumental in interpreting and reporting findings. RCG and SMA helped plan and interpret analyses of the barriers and facilitators items. CDH drafted the manuscript. All authors critically reviewed and contributed to the manuscript, and approved the final submission.

\section{Acknowledgements}

This work was supported by the Patient Centered Medical Home Demonstration Laboratory Coordination Center (XVA-61-041) from the United States (U.S.) Department of Veterans Affairs. The survey was conducted by the VA Healthcare Analysis and Information Group, and sponsored by the VA Office of Patient Care Services and Office of Clinical Operations. We are grateful to Drs. Gordon Schectman and Richard Stark, and Ms. Julie Kurutz for their support fielding the survey. The content does not represent the views of the U.S. Department of Veterans Affairs or the United States Government. 


\section{Author details}

Seattle-Denver Center of Innovation for Veteran-Centered and Value-Driven Care, VA Puget Sound, U.S. Department of Veterans Affairs, 1660 Columbian Way, S-152, Seattle, 98108 WA, USA. ${ }^{2}$ Department of Health Services, University of Washington School of Public Health, Seattle, WA, USA. ${ }^{3}$ Office of Analytics and Business Intelligence, U.S. Department of Veterans Affairs, Seattle, WA, USA. ${ }^{4}$ Center for Innovation to Implementation, VHA Palo Alto Healthcare System, Menlo Park, CA, USA. ${ }^{5}$ Center for Healthcare Organization and Implementation Research (CHOIR), VA Boston Healthcare System, Boston, MA, USA. 'Boston University School of Public Health, Boston, MA, USA. ${ }^{7}$ HSR\&D Center for the Study of Healthcare Innovation, Implementation, and Policy, VHA Greater Los Angeles Health Care System, North Hills, CA, USA. ${ }^{8}$ Department of Psychiatry and Biobehavioral Sciences, David Geffen School of Medicine, University of California, Los Angeles, CA, USA. ${ }^{9}$ Portland VHA Medical Center, VISN 20 Patient Aligned Care Team (PACT) Demonstration Laboratory, U.S. Department of Veterans Affairs, Portland, OR, USA. ${ }^{10}$ Center for Evaluation of Patient Aligned Care Teams (CEPACT), Philadelphia Veterans Affairs Medical Center, Philadelphia, USA. ${ }^{11}$ The Robert Wood Johnson Foundation Clinical Scholars Program, and the Department of Family and Community Medicine, Perelman School of Medicine of the University of Pennsylvania, Philadelphia, USA. ${ }^{12}$ Stanford University School of Medicine, Palo Alto, CA, USA. ${ }^{13}$ Department of Medicine, University of Washington School of Medicine, Seattle, WA, USA. ${ }^{14}$ RAND Corporation, Santa Monica, CA, USA. ${ }^{15}$ VA HSR\&D Center for the Study of Healthcare Innovation, Implementation, and Policy, Los Angeles, CA, USA.

\section{Received: 2 October 2015 Accepted: 17 February 2016}

\section{2.}

\section{References}

1. Peikes D, Zutshi A, Genevro J, Smith K, Parchman M, Meyers D. Early evidence on the patient-centered medical home. Rockville, MD: Agency for Healthcare Research and Quality. AHRQ Publication No. 12-0020-EF, 2012.

2. Alexander JA, Bae D. Does the patient-centred medical home work? A critical synthesis of research on patient-centred medical homes and patient-related outcomes. Health Serv Manage Res. 2012;25(2):51-9.

3. Hoff T, Weller W, DePuccio M. The patient-centered medical home: a review of recent research. Med Care Res Rev. 2012;69(6):619-44.

4. Jackson GL, Powers BJ, Chatterjee R, Prvu Bettger J, Kemper AR, Hasselblad $V$, et al. The patient-centered medical home: a systematic review. Ann Intern Med. 2013;158(3):169-78.

5. Edwards ST, Bitton A, Hong J, Landon BE. Patient-centered medical home initiatives expanded in 2009-13: providers, patients and payment incentives increased. Health Aff. 2014;33(10):1823-31.

6. Landon BE, Gill JM, Antonelli RC, Rich EC. Prospects for rebuilding primary care using the patient-centered medical home. Health Aff (Millwood). 2010;29(5):827-34

7. True G, Butler AE, Lamparska BG, Lempa ML, Shea JA, Asch DA, et al. Open access in the patient-centered medical home: lessons from the Veterans Health Administration. J Gen Intern Med. 2013;28(4):539-45.

8. Wagner EH, Gupta R, Coleman K. Practice transformation in the safety net medical home initiative: a qualitative look. Med Care. 2014;52:S18-22. doi:10.1097/MLR.0000000000000196.

9. Solimeo SL, Stewart KR, Stewart GL, Rosenthal G. Implementing a patient centered medical home in the Veterans health administration: perspectives of primary care providers. Healthcare. 2014;2(4): doi:10.1016/j.hjdsi.2014.07. 004.

10. Gale RC, Asch SM, Taylor T, Nelson KM, Luck J, Meredith LS, et al. The most used and most helpful facilitators for patient-centered medical home implementation. Implement Sci. 2015;10(1):52.

11. Helfrich C, Dolan E, Simonetti J, Reid R, Joos S, Wakefield B, et al. Elements of team-based care in a patient-centered medical home are associated with lower burnout among VA primary care employees. J Gen Intern Med. 2014:29(2):659-66.

12. Klein S. The Veterans Health Administration: implementing patient-centered medical homes in the nation's largest integrated delivery system. Commonwealth Fund publ. 2011;1537:16.

13. Nelson $\mathrm{K}$, Helfrich CD, Sun H, Hebert PL, Liu C-F, Dolan E, et al. Implementation of the Patient Centered Medical Home (PCMH) in the Veterans Health Administration (VHA); associations with patient satisfaction, provider burnout and utilization. JAMA Intern Med. 2014;174(8):1350-8.
14. Keenan PS, Normand S-LT, Lin Z, Drye EE, Bhat KR, Ross JS, et al. An administrative claims measure suitable for profiling hospital performance on the basis of 30-day all-cause readmission rates among patients with heart failure. Circulation. 2008;1(1):29-37.

15. Paustian ML, Alexander JA, El Reda DK, Wise CG, Green LA, Fetters MD. Partial and Incremental PCMH practice transformation: implications for quality and costs. Health Serv Res. 2014;49(1):52-74.

16. LeBreton JM, Senter JL. Answers to 20 questions about interrater reliability and interrater agreement. Organ Res Methods. 2008;11:815-52.

17. Rodriguez, H.P., L.S. Meredith, A.B. Hamilton, E.M. Yano, and L.V. Rubenstein, Huddle up!: the adoption and use of structured team communication for VA medical home implementation. Health Care Management Review, 2014. Publish Online Ahead of Print: p. 10.1097/HMR.0000000000000036.

18. Ladebue AC, Helfrich CD, Gerdes ZT, Finn SD, Nelson KM, Sayre GG. The experience of Patient Aligned Care Team (PACT) members. Health Care Management Review, 2014;41(1):2-10.

19. Sylling PW, Wong ES, Liu C-F, Hernandez SE, Batten AJ, Helfrich CD, et al. Patient-centered medical home implementation and primary care provider turnover. Med Care. 2014;52(12):1017-22.

20. Gilovich T, Griffin D, Kahneman D. Heuristics and biases: the psychology of intuitive judgment. Cambridge, UK: Cambridge University Press, 2002.

21. Patterson, B.J., S.L. Solimeo, K.R. Stewart, G.E. Rosenthal, P.J. Kaboli, and B.C Lund, Perceptions of pharmacists' integration into patient-centered medical home teams. Research in Social and Administrative Pharmacy, 2014. Available online ahead of print(0)

22. Alexander J, Cohen G, Wise C, Green L. The policy context of patient centered medical homes: perspectives of primary care providers. J Gen Intern Med. 2013;28(1):147-53.

\section{Submit your next manuscript to BioMed Central and we will help you at every step:}

- We accept pre-submission inquiries

- Our selector tool helps you to find the most relevant journal

- We provide round the clock customer support

- Convenient online submission

- Thorough peer review

- Inclusion in PubMed and all major indexing services

- Maximum visibility for your research

Submit your manuscript at www.biomedcentral.com/submit
) Biomed Central 